\title{
Blood Group-Tea Positive; Anti-Ageing Neurological Effects of Tea in Habitual Tea Drinkers
}

\author{
Divya $\mathrm{R}^{1 *}$ and Ashok $\mathrm{V}^{2}$ \\ ${ }^{1}$ Department of Physiology, Dhanalakshmi Srinivasan Medical college and Hospital (DSMCH), India \\ ${ }^{2}$ Biochemistry, Karpagam Faculty of medical sciences and research, India
}

*Corresponding author: Divya R, Associate Professor, Department of Physiology, Dhanalakshmi Srinivasan Medical college and Hospital (DSMCH), India

\section{Introduction}

Tea is a beverage made from the infusion of the leaves of Camellia synesis. A number of bioactive products such as theanine, tea polyphenols (TPP) and tea polysaccharides (TPS) are extracted from Tea. Studies show that the TPS acts as an antioxidant, anti- inflammatory agent, anticancer, antihyperglycemic properties [1]. The beneficial effects of tea are mainly due to catechin, L-theanine, and caffeine. Studies proves that catechin improves cognition, working memory and memory recognition [2].

\section{Ageing and Brain}

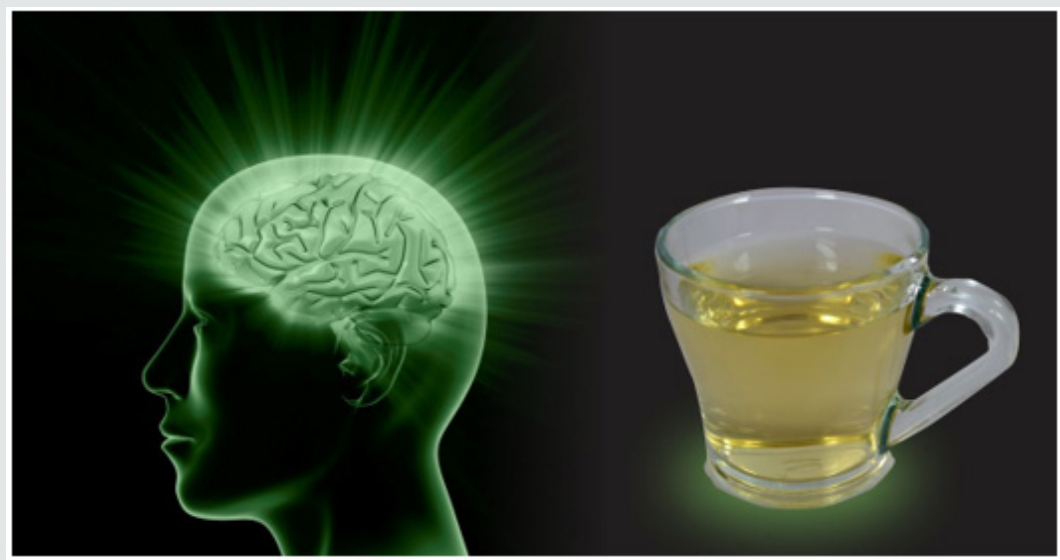

Figure 1. A slower ageing brain may hold more number of interconnections between regions with lesser commotion in connectivity.

Studies suggests that ageing related brain alterations results in reduced interregional connectivity in frontal cortex leading to reduced efficacy for intercommunication and transmission of information between the neuronal areas [3]. It also leads to leftward hemispheric asymmetry in structural connectivity [4]. Ageing results in a neurodegenerative brain with reduced functional connectivity in the DMN (Default mode network) during rest, which presents as cognitive decline and decreased task implementation. A slower ageing brain may hold more number of interconnections between regions with lesser commotion in connectivity [3] (Figure 1).

\section{Effects of Tea on Structural Brain Networks}

Recent research suggests that in geriatric age group habitual tea drinking had a greater functioning interregional connectivity and more efficient structural networking. Tea drinking also had a regional effect such as increased nodal efficiency. It also leads to 
suppression of alterations towards leftward asymmetry resulting in a pattern analogous to the rightward asymmetry which is found in middle age. This results in a better visuospatial functioning and information processing. During rest, the regions in the DMN (Default mode network) are found to be active, they remain unified resulting in better task implementation in the tea drinking group [3].

\section{Conclusion}

A simple lifestyle choice like tea drinking benefits brain health by slowing the cognitive decline due to ageing.

\section{References}

1. Du LL, Fu QY, Xiang LP (2016) Tea Polysaccharides and Their Bioactivities. Molecules 21(11): 1449.

2. National University of Singapore (2019) Drinking tea improves brain health, study suggests. Science Daily.

3. Junhua Li, Rafael Romero-Garcia, John Suckling, Lei Feng (2019) Habitual tea drinking modulates brain efficiency: evidence from brain connectivity evaluation. Aging 11(11): 3876.

4. Caeyenberghs K, Leemans A (2014) Hemispheric lateralization of topological organization in structural brain networks. Hum Brain Mapp 35: 4944-4957.

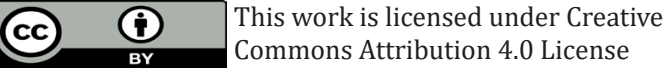

To Submit Your Article Click Here: Submit Article

DOI: $10.32474 /$ GJAPM.2019.02.000136

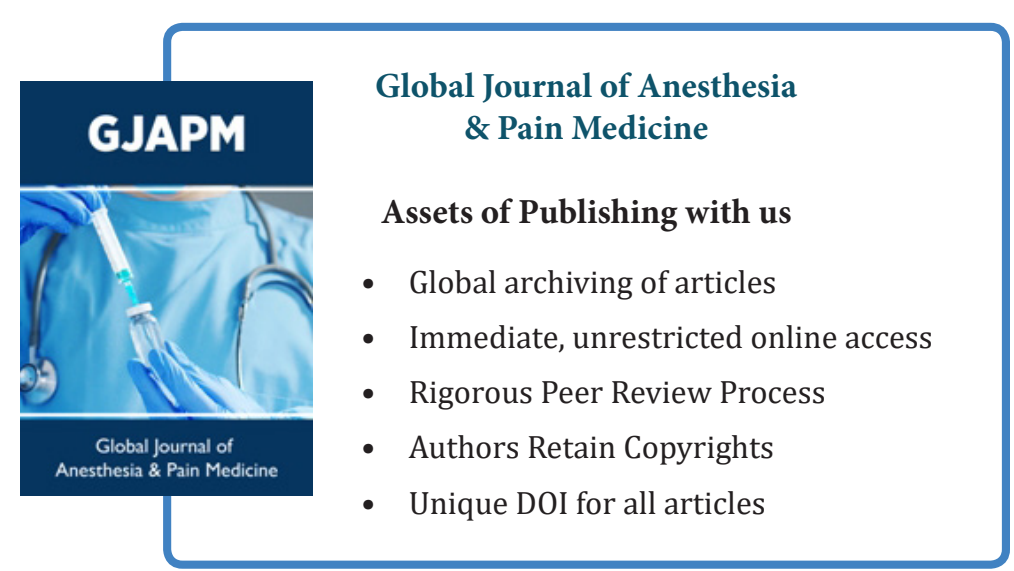

